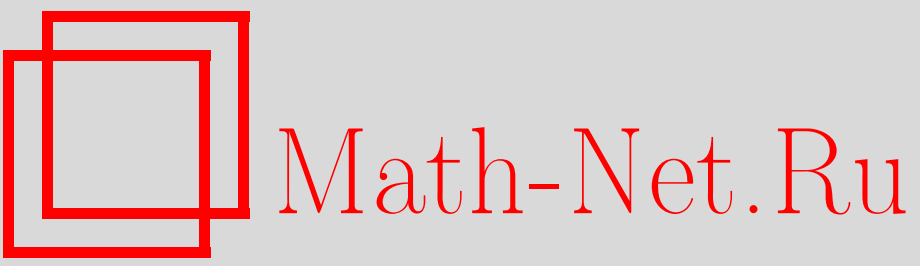

Г. И. Ивченко, Ю. И. Медведев, В. А. Миронова, Симметрические булевы функции и их метрические свойства, Матем. вопр. криптогр., 2013, том 4, выпуск 4, 49-63

DOI: https://doi.org/10.4213/mvk99

Использование Общероссийского математического портала Math-Net.Ru подразумевает, что вы прочитали и согласны с пользовательским соглашением

http: //www . mathnet.ru/rus/agreement

Параметры загрузки:

IP : 52.6 .47 .48

26 апреля 2023 г., 14:07:21 
УДК 519.212.2

\title{
Симметрические булевы функции и их метрические свойства
}

\author{
Г. И. Ивченко ${ }^{1}$, Ю. И. Медведев ${ }^{2}$, В. А. Миронова ${ }^{3}$ \\ ${ }^{1,3}$ НИУ Высшая школа экономики, Москва \\ ${ }^{2}$ Академия криптографии Российской Федерации, Москва
}

Получено 22.IV.2013

Работа посвящена анализу (в том числе и в стохастической постановке) различных метрических свойств симметрических булевых функций: находятся расстояния от заданной булевой функции до ближайшей к ней и наиболее удаленной от нее симметрических функций, а также до их подмножеств, исследуются структура и объем множества булевых функций, наиболее удаленных от класса симметрических функций, и сопутствующие вопросы.

Ключевые слова: симметрическая булева функция, вектор уровней, расстояние Хэмминга, стохастическая модель, биномиальное отклонение, предельные теоремы, $S$-функции

Symmetric Boolean functions and their metric properties matrices of transitions of differences when using some modular groups

\section{G. I. Ivchenko ${ }^{1}$, Yu. I. Medvedev ${ }^{2}$, V. A. Mironova ${ }^{3}$ \\ ${ }^{1,3} \mathrm{NRU}$ Higher School of Economics, Moscow \\ ${ }^{2}$ Academy of Cryptography of the Russian Federation, Moscow}

\begin{abstract}
Various metric properties of symmetric Boolean functions are analysed (including the case of random functions). The minimal and maximal distances from a given Boolean function to the set of symmetric functions (as well to its subsets) are found. The structure and the size of the set of functions which are the farthest from the symmetric functions set are investi-gated.
\end{abstract}

Key words: symmetric Boolean function, level vector, Hamming distance, probabilistic model, binomial deviation, limit theorems, $S$-functions

Citation: Mathematical Aspects of Cryptography, 2013, vol. 4, no. 4, pp. 49-63 (Russian). 


\section{1. Введение}

Пусть $V_{n}=\{0,1\}^{n}-n$-мерное векторное пространство над полем из двух элементов, $f: V_{n} \rightarrow\{0,1\}-$ булева функция от $n$ переменных, $F_{n}-$ множество всех таких функций и $S_{n} \subset F_{n}$ - подмножество симметрических функций, т. е. таких функций, которые принимают одно и то же значение на всех векторах одинакового веса (или, более традиционно, которые не меняются при любой перестановке их аргументов). Симметрические булевы функции (с.б.ф.) находят широкое применение в теории кодирования, в криптографической теории и практике, и их исследованию посвящена обширная литература, обзор которой можно найти в $[1,2,3]$ (см. также $[4,6$, 7]). Их важной особенностью является экономность задания с.б.ф. в виде булева вектора размерности $n+1$, вместо $2^{n}$-мерного булева вектора (таблицы истинности) для произвольной б.ф. $f \in F_{n}$ (так что их число $\left|S_{n}\right|=2^{n+1}$, в то время как $\left|F_{n}\right|=2^{2^{n}}$ ). С другой стороны, симметрические булевы функции обладают рядом важных свойств, и их используют в качестве аппроксимаций для произвольных булевых функций. В этом аспекте важное значение имеют метрические свойства с.б.ф., анализу которых и посвящена данная работа. Мы рассматриваем эти вопросы также и в стохастической постановке, когда на множестве $F_{n}$ вводится вероятностная мера, в соответствии с которой все значения случайной б.ф. $f \in F_{n}$ представляют собой совокупность независимых одинаково распределенных бернуллиевских случайных величин.

Напомним предварительно некоторые сведения о булевых (а также симметрических) функциях.

Весом $\|x\|$ булева вектора $x$ (весом $\|f\|$ булевой функции $f$ ) называется число его единичных координат (число ее единичных значений), и расстоянием Хэмминга $\rho(f, g)$ между булевыми функциями $f$ и $g$ называется вес $\|f \oplus g\|$ функции $f \oplus g$ (здесь $\oplus-$ знак сложения по $\bmod 2$ ): $\rho(f, g)=\|f \oplus g\|$.

Поскольку с.б.ф. $f$ принимает одно и то же значение на всех векторах одинакового веса (уровня) $\|x\|$, то она может быть задана вектором

$$
s=s(f)=\left(s_{0}, s_{1}, \ldots, s_{n}\right),
$$


где $s_{j}=f(x)$ при $\|x\|=j, \quad j=0,1, \ldots, n$, который называется ее вектором уровней.

Напомним также, что любая с.б.ф. $f \in S_{n}$ представима в виде

$$
f(x)=\sum_{j=0}^{n} \lambda_{j} \sigma_{j}(x)
$$

где $\sigma_{j}(x)$ - элементарная симметрическая функция:

$$
\sigma_{j}(x)=\underset{1 \leq i_{1}<i_{2}<\ldots<i_{j} \leq n}{\bigoplus} x_{i_{1}} x_{i_{2}} \ldots x_{i_{j}} .
$$

Булев вектор

$$
\lambda=\lambda(f)=\left(\lambda_{0}, \lambda_{1}, \ldots, \lambda_{n}\right)
$$

называется простым вектором алгебраической нормальной формы с.б.ф. $f$.

Между векторами (1) и (2) имеет место следующая связь:

$$
\lambda_{i}=\sum_{j \leq i} s_{j}, \quad s_{i}=\sum_{j \leq i} \lambda_{j} .
$$

Здесь символ $j \preceq i$ означает, что $j$ предшествует $i$, т. е. при представлении целых чисел $i$ и $j$ в двоичной форме:

$$
\begin{aligned}
& i=i_{0}+i_{1} 2+i_{2} 2^{2}+\ldots+i_{k} 2^{k}, \\
& j=j_{0}+j_{1} 2+j_{2} 2^{2}+\ldots+j_{m} 2^{m}, i_{0}, \ldots, i_{k}, j_{0}, \ldots, j_{m} \in\{0,1\},
\end{aligned}
$$

для всех коэффициентов должны выполняться неравенства $j_{s} \leq i_{s}, s=0,1, \ldots$.

В дальнейшем нам понадобится одно арифметическое свойство биномиальных коэффициентов $\left(\begin{array}{l}n \\ k\end{array}\right), k=0,1, \ldots n$. Известная теорема Лукача [8] утверждает, что если $p-$ простое число и $n=\sum_{i=0}^{s} n_{i} p^{i}, \quad k=\sum_{i=0}^{s} k_{i} p^{i}-$ $p$-е представления чисел $n$ и $k$, то $\left(\begin{array}{l}n \\ k\end{array}\right) \equiv \prod_{i=0}^{s}\left(\begin{array}{l}n_{i} \\ k_{i}\end{array}\right)(\bmod p)$.

В случае $p=2$ отсюда следует, что $\left(\begin{array}{l}n \\ k\end{array}\right) \equiv 1(\bmod 2)$ тогда и только тогда, когда $k \preceq n$. Это, в свою очередь, означает, что при заданном $n$ число 
нечетных биномиальных коэффициентов есть $\alpha(n)=2^{\sum_{i n_{i}}}, \quad$ где $n=\sum_{i} n_{i} 2^{i}-$ разложение по степеням двойки.

В частности, если $n=2^{s}, s \geq 1$, то все биномиальные коэффициенты, за исключением двух крайних (которые всегда нечетные), четные, а если $n=2^{s}-1, s \geq 2$, то все они - нечетные.

Дальнейшие детали этой темы приведены в приложении.

\section{2. Метрические свойства симметрических булевых функций}

1) Расстояние между двумя с.б.ф. Расстояние $\rho\left(f_{1}, f_{2}\right)$ между двумя произвольными с.б.ф. $f_{1}$ и $f_{2}$ вычисляется весьма просто через их векторы уровней $s^{(i)}=s\left(f_{i}\right)=\left(s_{0}^{(i)}, s_{1}^{(i)}, \ldots, s_{n}^{(i)}\right), i=1,2$, следующим образом. Пусть векторы $s^{(1)}$ и $s^{(2)}$ различаются лишь на уровнях $k_{1}, k_{2}, \ldots, k_{s}$, $0 \leq k_{1}<k_{2}<\ldots<k_{s} \leq n, 1 \leq s \leq n$. Тогда, очевидно,

$$
\rho\left(f_{1}, f_{2}\right)=\left\|f_{1} \oplus f_{2}\right\|=\left(\begin{array}{l}
n \\
k_{1}
\end{array}\right)+\ldots+\left(\begin{array}{l}
n \\
k_{s}
\end{array}\right) .
$$

Одной из интересных в теоретическом и прикладном аспектах задач является задача оценки расстояния от заданной с.б.ф. $f$ до некоторого подмножества $A \subseteq S_{n}: \min _{g \in A}\|f \oplus g\|$, где в качестве $A$ может быть взято, например, подмножество $S_{n k}$ функций степени, не превышающей $k, 1 \leq k \leq n$, т. е. когда в (2) все $\lambda_{j}=0$ при $j>k\left(S_{n k}=S_{n}\right.$ при $\left.k=n\right)$.

2) Расстояние между произвольными б.ф. и с.б.ф. Найдем теперь расстояние $\rho(f, s)$ между произвольными б.ф. $f$ и с.б.ф. $s \in S_{n}$. Пусть $f_{j}$ обозначает вес функции $f(x)$ на уровне $\|x\|=j$ :

$$
f_{j}=\sum_{x \in V_{n}} f(x) I(\|x\|=j), \quad j=0,1, \ldots, n
$$

(здесь и далее $I(\cdot)$ - индикатор). Пусть также

$$
b_{j}=b_{j}(n)=\left(\begin{array}{c}
n \\
j
\end{array}\right)-2 f_{j}, \quad j=0,1, \ldots, n .
$$


Поскольку число векторов $x$ веса $j$ равно $\left(\begin{array}{l}n \\ j\end{array}\right)$, а для булевых переменных $a$ и $b$ выполняется равенство $a \oplus b=a+b-2 a b$, то можно записать следующую цепочку соотношений (в ней и далее $\left(s_{0}, s_{1}, \ldots, s_{n}\right)$ - вектор уровней с.б.ф. $s$ ):

$$
\begin{aligned}
& \rho(f, s)=\|f(x) \oplus s(x)\|=\sum_{j=0}^{n} \sum_{x \in V_{n}}[f(x)+s(x)-2 f(x) s(x)] I(\|x\|=j)= \\
& =\sum_{j=0}^{n}\left[f_{j}+\left(\begin{array}{c}
n \\
j
\end{array}\right) s_{j}-2 f_{j} s_{j}\right]=\|f\|+\sum_{j=0}^{n} b_{j} s_{j}=\frac{1}{2} \sum_{j=0}^{n}\left[\left(\begin{array}{l}
n \\
j
\end{array}\right)-b_{j}\right]+\sum_{j=0}^{n} b_{j} s_{j}= \\
& =2^{n-1}-\frac{1}{2} \sum_{j=0}^{n} b_{j}\left(1-2 s_{j}\right)=2^{n-1}-\frac{1}{2} \sum_{j=0}^{n} b_{j}(-1)^{s_{j}} .
\end{aligned}
$$

В частности, если $f \in S_{n k}$, то $s_{j}=0$ при $j>k$, и формула (4) принимает в этом случае вид:

$$
\rho(f, s)=\|f\|+\sum_{j=0}^{k} b_{j} s_{j}=2^{n-1}-\frac{1}{2} \sum_{j=0}^{k} b_{j}(-1)^{s_{j}}-\frac{1}{2} \sum_{j=k+1}^{n} b_{j}
$$

(при $k=n$ последняя сумма заменяется нулем).

3) Расстояние до подмножества. Обозначим через

$$
\rho_{n k}=\rho\left(f, S_{n k}\right)=\min _{s \in S_{n k}} \rho(f, s)
$$

расстояние от б.ф. $f$ до подмножества $S_{n k}$.

Из формулы (5) непосредственно следует, что этот минимум достигается, если $s_{j}=I\left(b_{j}<0\right)$ для всех $j=0,1, \ldots, k$, и в этом случае

$$
\rho_{n k}=\|f\|+\sum_{j=0}^{k} b_{j} I\left(b_{j}<0\right)=2^{n-1}-\frac{1}{2} \sum_{j=0}^{k}\left|b_{j}\right|-\frac{1}{2} \sum_{j=k+1}^{n} b_{j} .
$$

Если же в (5) для некоторых $j$ значение $b_{j}=0$, то соответствующие $s_{j}$ можно задать произвольно: 0 или 1 , и если

$$
\mu_{n k}=\sum_{j=0}^{k} I\left(b_{j}=0\right)=\sum_{j=0}^{k} I\left(2 f_{j}=\left(\begin{array}{c}
n \\
j
\end{array}\right)\right),
$$

то множество $M_{n k}$ ближайших к $f$ с.б.ф. $s \in S_{n k}$ имеет объем $2^{\mu_{n k}}$. 
Для случая $k=n$ формула (6) дает важный самостоятельный результат: расстояние от б.ф. $f$ до множества всех с.б.ф. $S_{n}$ есть

$$
\rho_{n}=\rho\left(f, S_{n}\right)=\min _{s \in S_{n}} \rho(f, s)=2^{n-1}-\frac{1}{2} \sum_{j=0}^{n}\left|b_{j}\right| .
$$

4) Максимальное расстояние. Пусть теперь

$$
\delta_{n k}=\max _{s \in S_{n k}} \rho(f, s) .
$$

Из (5) следует, что максимально удаленная от $f$ с.б.ф. $s \in S_{n k}$ задается координатами уровней $s_{j}=I\left(b_{j}>0\right)$ для всех $j=0,1, \ldots, k$, и тогда

$$
\delta_{n k}=\|f\|+\sum_{j=0}^{k} b_{j} I\left(b_{j}>0\right)=2^{n-1}+\frac{1}{2} \sum_{j=0}^{k}\left|b_{j}\right|-\frac{1}{2} \sum_{j=k+1}^{n} b_{j} .
$$

В частности, при $k=n$ имеем

$$
\delta_{n}=\max _{s \in S_{n}} \rho(f, s)=2^{n-1}+\frac{1}{2} \sum_{j=0}^{n}\left|b_{j}\right| .
$$

Таким образом, для любой с.б.ф. $s \in S_{n k}$ ее расстояние от заданной б. ф. $f$ удовлетворяет неравенствам

$$
\sum_{j=0}^{k} b_{j} I\left(b_{j}<0\right) \leq \rho(f, s)-\|f\| \leq \sum_{j=0}^{k} b_{j} I\left(b_{j}>0\right) .
$$

\section{3. Стохастическая модель: распределения метрических характеристик}

1) Постановка задачи. Пусть на множестве $F_{n}$ задана вероятностная мера $\mathbf{P}$, в соответствии с которой все значения произвольной б.ф. $f \in F_{n}$ представляют собой совокупность независимых бернуллиевских случайных величин (с.в.) и при этом для любого $x \in V_{n}$

$$
\mathbf{P}\{f(x)=1\}=1-\mathbf{P}\{f(x)=0\}=p=1-q .
$$

В этом случае веса $f_{j}, j=0,1, \ldots, n$, такой случайной функции будут независимыми в совокупности биномиальными случайными величинами

$$
\mathscr{L}\left(f_{j}\right)=\operatorname{Bi}\left(a_{n j}, p\right), \quad a_{n j}=\left(\begin{array}{c}
n \\
j
\end{array}\right), \quad j=0,1, \ldots, n
$$

(эти обозначения используются везде в дальнейшем). 
Как следует из предыдущего, в обсуждаемой проблематике ключевую роль играют величины (см. соотношения (6) - (9)) $\xi_{n j}=\left|b_{j}\right|=\left|a_{n j}-2 f_{j}\right|$, которые мы будем называть биномиальными отклонениями (для равномерной модели, т. е. при $p=1 / 2$, это будут отклонения биномиальных с.в. от своих средних значений). Исследуем сначала распределения этих с.в., при этом далее будем предполагать, что $1 \leq j \leq n-1$, так как, очевидно, $\xi_{n 0}=\xi_{n n}=1$.

Предварительно мы установим некоторые общие свойства биномиального отклонения, представляющие и самостоятельный интерес.

2) Распределение биномиального отклонения. Пусть $X_{N}$ есть биномиальная с.в. $\mathscr{L}\left(X_{N}\right)=\operatorname{Bi}(N, p)$, и $Y_{N}=\left|N-2 X_{N}\right|$ - ее биномиальное отклонение. Используя стандартные обозначения для биномиальных вероятностей $b(m ; N, p)=\left(\begin{array}{l}N \\ m\end{array}\right) p^{m} q^{N-m}$, и функции распределения $B(k ; N, p)=$ $=\sum_{m=0}^{k} b(m ; N, p)$, несложно выписать явный вид распределения и моментов этой с.в.

Утверждение 1. Распределение с.в. $Y_{N}$ сосредоточено на решетке неотрицательных иелых чисел четности, одинаковой с четностью числа $N$, именно: если $N=2 s$, то

$$
\begin{aligned}
& \mathbf{P}\left\{Y_{N}=2 r\right\}=b(s-r ; N, p)+b(s+r ; N, p), r=1, \ldots, s, \\
& \mathbf{P}\left\{Y_{N}=0\right\}=b(s ; N, p) ;
\end{aligned}
$$

если $N=2 s+1$, то

$$
\mathbf{P}\left\{Y_{N}=2 r+1\right\}=b(s-r ; N, p)+b(s+r ; N, p), r=0,1, \ldots, s .
$$

Для первых двух моментов справедливы представления

$$
\begin{gathered}
\mathbf{E} Y_{N}=N \delta(B(c ; N, p)-B(c ; N, q))+ \\
\frac{N}{2}\left(1-\delta^{2}\right)(b(c ; N-1, p)+b(c ; N-1, q)), \\
\mathbf{E} Y_{N}^{2}=N^{2} \delta^{2}+N\left(1-\delta^{2}\right),
\end{gathered}
$$

где $\delta=q-p, \quad c=[N / 2]$ - иелая часть $N / 2$. 
Доказательство. Вид распределения следует из очевидных соотношений: при $m \geq 1$

$$
\begin{aligned}
& \mathbf{P}\left\{Y_{N}=m\right\}=\mathbf{P}\left\{X_{N}=\frac{N-m}{2}\right\}+\mathbf{P}\left\{X_{N}=\frac{N+m}{2}\right\}= \\
& =\left(b\left(\frac{N-m}{2} ; N, p\right)+b\left(\frac{N+m}{2} ; N, p\right)\right) I(N-m \equiv 0(\bmod 2))
\end{aligned}
$$

и

$$
\mathbf{P}\left\{Y_{N}=0\right\}=\mathbf{P}\left\{X_{N}=\frac{N}{2}\right\}=b\left(\frac{N}{2} ; N, p\right) I(N \equiv 0(\bmod 2)) .
$$

Формула для математического ожидания получается непосредственно из определения с использованием известного рекуррентного соотношения

$$
B(k-1 ; N-1, p)=B(k ; N, p)-q b(k ; N-1, p),
$$

а второй момент - прямое следствие формул для моментов биномиальной с.в.

Отметим важный частный случай: для равномерной модели, т. е. при $p=1 / 2 \Leftrightarrow \delta=0$,

$$
\begin{gathered}
\mathbf{E} Y_{N}=N b\left(c ; N-1, \frac{1}{2}\right)=N\left(\begin{array}{c}
N-1 \\
{[N / 2]}
\end{array}\right) 2^{-N+1}, \\
\mathbf{D} Y_{N}=N\left(1-N b^{2}\left(c ; N-1, \frac{1}{2}\right)\right) .
\end{gathered}
$$

Приведем еще вид четвертого момента (рутинные выкладки мы опускаем):

$$
\mathbf{E} Y_{N}^{4}=N^{4} \delta^{4}+6 N^{3} \delta^{2}\left(1-\delta^{2}\right)+N^{2}\left(1-\delta^{2}\right)\left(3-11 \delta^{2}\right)+2 N\left(1-\delta^{2}\right)\left(3 \delta^{2}-1\right) .
$$

Дополним проведенный анализ еще и исследованием асимптотического поведения с.в. $Y_{N}$ в классических условиях: $N \rightarrow \infty$, параметр $p \in(0,1)$ фиксирован. Применяя к с.в. $X_{N}$ теорему Муавра-Лапласа (как локальный, так и интегральный ее варианты), а также некоторые известные факты теории больших уклонений для биномиального распределения, технически несложно установить следующий результат.

Утверждение 2. Пусть $N \rightarrow \infty$, a $p \in(0,1)$ фиксировано. Тогда при $\delta=q-p \neq 0$ с.в. $Y_{N}$ асимптотически нормальна с параметрами

$$
\mathbf{E} Y_{N} \sim N|\delta|, \quad \mathbf{D} Y_{N} \sim N\left(1-\delta^{2}\right),
$$


a при $\delta=0$ ее распределение удовлетворяет асимптотическому соотношению

$$
\mathscr{L}\left(Y_{N} / \sqrt{N}\right) \sim \mathscr{L}(|\xi|),
$$

где $\xi-$ стандартная нормальная с.в., при этом

$$
\mathbf{E} Y_{N} \sim \sqrt{\frac{2}{\pi} N}, \quad \mathbf{D} Y_{N} \sim \frac{\pi-2}{\pi} N .
$$

Доказательство. Функция распределения с.в. $Y_{N}$ имеет вид

$$
\begin{aligned}
& \mathbf{P}\left\{Y_{N} \leq m\right\}=\mathbf{P}\left\{\frac{N-m}{2} \leq X_{N} \leq \frac{N+m}{2}\right\}= \\
& =B\left(\frac{N+m}{2} ; N, p\right)-B\left(\frac{N-m}{2}-1 ; N, p\right) .
\end{aligned}
$$

Пусть $p<q$. Выбирая $m$ в виде

$$
m=N \delta+2 x \sqrt{N p q}, \quad|x| \leq C<\infty,
$$

будем иметь

$$
\begin{aligned}
& \left(\frac{N+m}{2}-N p\right) / \sqrt{N p q}=\delta \sqrt{\frac{N}{p q}}+x \rightarrow \infty, \\
& \left(\frac{N-m}{2}-N p\right) / \sqrt{N p q}=-x .
\end{aligned}
$$

Следовательно, первая биномиальная сумма в правой части (13) стремится к 1 , а вторая - к $\Phi(-x)$, где $\Phi-$ стандартная нормальная функция распределения, что в итоге дает

$$
\mathbf{P}\left\{\frac{Y_{N}-N \delta}{2 \sqrt{N p q}} \leq x\right\} \rightarrow 1-\Phi(-x)=\Phi(x)
$$

или (так как $\left.4 p q=1-\delta^{2}\right) \mathscr{L}\left(Y_{N}\right) \sim \mathcal{N}\left(N \delta, N\left(1-\delta^{2}\right)\right)$.

Аналогично анализируется случай $p>q$.

Наконец, при $p=q$ с.в. $X_{N}$ асимптотически нормальна $\mathcal{N} \mathcal{N}(N / 2, N / 4)$ или, что то же, $\mathscr{L}\left(\left(2 X_{N}-N\right) / \sqrt{N}\right) \sim \mathcal{N}(0,1)$, что эквивалентно соответствующей формулировке утверждения.

Что касается асимптотик моментов, то они непосредственно следуют из точных формул (11)-(12) с учетом того факта, что скорость убывания как 
локальных вероятностей, так и "хвостов" биномиального распределения в зонах больших уклонений экспоненциально быстрая (см., например, [5]).

Замечания. 1 . Для с.в. $Y_{N}$ имеют место также и соответствующие утверждения о локальной сходимости.

2. Пусть $Y_{N}(a)=\left|N-a X_{N}\right|, a>1$. Тогда аналог утверждения 2 для этой с.в. имеет следующий вид: при $\delta=1-a p \neq 0$

$$
\mathscr{L}\left(Y_{N}(a)\right) \sim \mathcal{N} \mathcal{N}\left(N|\delta|, N a^{2} p q\right),
$$

если же $\delta=0\left(a=p^{-1}\right)$, то $\mathscr{L}\left(Y_{N}\left(p^{-1}\right) / \sqrt{N q / p}\right) \sim \mathscr{L}(|\xi|)$.

3) Статистический критерий. Из утверждения 2 следует, что распределение биномиального отклонения $Y_{N}$ весьма "чувствительно" к отклонениям от равномерности: при $p=1 / 2 \Leftrightarrow \delta=0$ оно асимптотически сосредоточено в окрестности точки $\sqrt{2 N / \pi}$, а при $\delta \neq 0-$ в окрестности точки $N|\delta|$, и эти окрестности асимптотически не пересекаются (так как имеют размеры порядка $\sqrt{N}$ ). Это подсказывает идею использовать величину $Y_{N}$ в качестве новой тестовой статистики для проверки гипотезы $H_{0}: p=1 / 2$ в биномиальной модели $\operatorname{Bi}(N, p)$, когда $N \rightarrow \infty$. Именно, если задать критическую область в виде

$$
\mathrm{X}_{t}=\left\{Y_{N}>N^{t}\right\}, \quad 1 / 2<t<1,
$$

то в силу утверждения 2 ошибки первого и второго рода такого критерия будут вести себя при $N \rightarrow \infty$ следующим образом (ниже альтернатива обозначена как $\left.H_{\delta}: q-p=\delta \neq 0\right)$ :

$$
\begin{aligned}
& \alpha_{N t}=\mathbf{P}\left\{Y_{N}>N^{t} \mid H_{0}\right\}=\mathbf{P}\left\{Y_{N} / \sqrt{N}>N^{t-1 / 2} \mid H_{0}\right\} \rightarrow 0, \\
& \beta_{N t}=\mathbf{P}\left\{Y_{N} \leq N^{t} \mid H_{\delta}\right\}=\mathbf{P}\left\{\left(Y_{N}-N|\delta|\right) / \sqrt{N} \leq N^{t-1 / 2}-\sqrt{N}|\delta| \mid H_{\delta}\right\} \rightarrow 0,
\end{aligned}
$$

поскольку $N^{t-1 / 2} \rightarrow \infty$, а $N^{t-1 / 2}-\sqrt{N} \rightarrow-\infty$.

Таким образом, при любом $t \in(1 / 2,1)$ критерий (14) асимптотически безошибочно различает гипотезы $H_{0}$ и $H_{\delta}$ для любого $\delta \neq 0$.

4) Распределения метрических характеристик. Вернемся к с.в. $\xi_{n j}=$ $=\left|b_{j}\right|=\left|a_{n j}-2 f_{j}\right|$. Для всех $j=1, \ldots, n-1 \quad$ биномиальные коэффициенты $a_{n j} \rightarrow \infty$, когда $n \rightarrow \infty$, поэтому ко всем этим величинам применимы полу- 
ченные выше результаты для биномиального уклонения с заменой $N$ на $a_{n j}$ для с.в. $\xi_{n j}$, что позволяет исследовать асимптотическое (при $n \rightarrow \infty$ ) поведение основных метрических характеристик $\rho_{n k}$ (см. (6)) и $\delta_{n k}$ (см. (8)).

Рассмотрим более детально наиболее важный случай $k=n$ (случай произвольного $k$ рассматривается аналогично). Обозначим

$$
\eta_{n}=\sum_{j=1}^{n-1} \xi_{n j}
$$

В этой сумме слагаемые независимы и при $\delta=q-p \neq 0$, в силу утверждения 2 они асимптотически нормальны с параметрами $\left(a_{n j}|\delta|, a_{n j}\left(1-\delta^{2}\right)\right)$ для $j$-го слагаемого. Следовательно, вся эта сумма асимптотически нормальна с параметрами $\left(2^{n}|\delta|, 2^{n}\left(1-\delta^{2}\right)\right)$.

Если же $\delta=0$, то с.в. $\xi_{n j}$ асимптотически ведет себя как с.в. $\xi_{n j}^{*}=\sqrt{a_{n j}}\left|\xi_{j}\right|$, где $\xi_{j}-$ стандартная нормальная с.в. Для совокупности этих с.в. нетрудно оценить следующую дробь Ляпунова:

$$
\sum_{j=1}^{n-1} \mathbf{E}\left(\xi_{n j}^{*}-\mathbf{E} \xi_{n j}^{*}\right)^{4} /\left(\sum_{j=1}^{n-1} \mathbf{D} \xi_{n j}^{*}\right)^{2},
$$

она, очевидно, имеет порядок

$$
\sum_{j=1}^{n-1} a_{n j}^{2} /\left(\sum_{j=1}^{n-1} a_{n j}\right)^{2} \sim\left(\begin{array}{c}
2 n \\
n
\end{array}\right) 2^{-2 n} \sim(\pi n)^{-1 / 2},
$$

т. е. стремится к 0 при $n \rightarrow \infty$.

Следовательно, к их сумме и в этом случае применима центральная предельная теорема, что приводит к заключению

$$
\mathscr{L}\left(\eta_{n}\right) \sim \mathcal{N}\left(\sqrt{\frac{2}{\pi}} C_{n}, \frac{\pi-2}{\pi} 2^{n}\right), \quad C_{n}=\sum_{j=0}^{n}\left(\begin{array}{l}
n \\
j
\end{array}\right)^{1 / 2} .
$$

В итоге можно сформулировать следующее утверждение.

Теорема. Если $n \rightarrow \infty$, а $p \in(0,1)$ фиксировано, то

$$
\mathscr{L}\left(\rho_{n}\right) \sim\left\{\begin{array}{lll}
\mathcal{N}\left((1-|\delta|) 2^{n-1},\left(1-\delta^{2}\right) 2^{n-2}\right) & \text { при } & p \neq \frac{1}{2}, \\
\mathcal{N}\left(2^{n-1}-\frac{1}{\sqrt{2 \pi}} C_{n}, \frac{\pi-2}{\pi} 2^{n-2}\right) & \text { при } & p=\frac{1}{2},
\end{array}\right.
$$




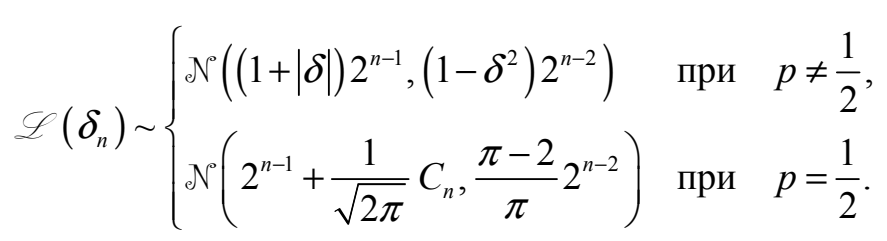

\section{4. $S$-функции}

1. Назовем булеву функцию $f \in F_{n} S$-фуннциией, если она максимально удалена от класса $S_{n}$ симметрических функций, т. е. на ней достигается

$$
r_{n}=\max _{f \in F_{n}} \rho\left(f, S_{n}\right)
$$

а множество всех $S$-функций обозначим $R_{n}$.

Основой для нахождения и описания таких функций является соотношение (7), из которого следует, что нужно найти как

$$
\min \sum_{j=0}^{n}\left|b_{j}\right|=\min \sum_{j=0}^{n}\left|a_{n j}-2 f_{j}\right|, \quad a_{n j}=\left(\begin{array}{c}
n \\
j
\end{array}\right),
$$

так и те функции, на которых этот минимум достигается. Очевидно, что минимум достигается при $f_{j}=a_{n j} / 2$, если число $a_{n j}$ четное, и при $f_{j}=\left(a_{n j} \pm 1\right) / 2$, если $a_{n j}$ нечетное, что можно записать в виде

$$
f_{j}=\left[a_{n j} \pm I\left(a_{n j} \equiv 1(\bmod 2)\right)\right] / 2 .
$$

Сам же минимум (15) равен при этом числу $\alpha(n)$ нечетных биномиальных коэффициентов $a_{n j}$ (см. введение).

Следовательно,

$$
r_{n}=\max _{f \in F_{n}} \rho\left(f, S_{n}\right)=2^{n-1}-\frac{\alpha(n)}{2} .
$$

Замечание. Свойства величины $\alpha(n)$ и вытекающие из них оценки для $r_{n}$ приведены в приложении.

2. Опишем теперь структуру функций $f \in R_{n}$ и найдем их число $N_{n}$.

Рассмотрим таблицу истинности произвольной $S$-функции. Если число $a_{n j}$ четное, то в ее строках, соответствующих векторам $x$ веса $\|x\|=j$, следует положить половину значений функции равными 1 и половину зна- 
чений равными 0: число различных вариантов в этом случае равно $\left(\begin{array}{c}a_{n j} \\ a_{n j} / 2\end{array}\right)$.

Если же число $a_{n j}$ нечетное, то число значений функции при $\|x\|=j$ равных 1 следует взять равным либо $\left(a_{n j}+1\right) / 2$, либо $\left(a_{n j}-1\right) / 2$; число различных вариантов в этом случае равно $2\left(\begin{array}{c}a_{n j} \\ \left(a_{n j}+1\right) / 2\end{array}\right)$.

Отсюда следует, что объем множества $R_{n}$ есть

$$
N_{n}=\left|R_{n}\right|=\sum_{j: a_{n j} \text {-чет }}\left(\begin{array}{c}
a_{n j} \\
a_{n j} / 2
\end{array}\right)+2 \sum_{j: a_{n j} \text {-иечет }}\left(\begin{array}{c}
a_{n j} \\
\left(a_{n j}+1\right) / 2
\end{array}\right) .
$$

\section{Приложение. Арифметические свойства биномиальных коэффициентов}

1. Как отмечено во введении, для числа $\alpha(n)$ нечетных биномиальных коэффициентов $a_{n j}$ имеет место представление

$$
\alpha(n)=\sum_{j=0}^{n} I\left(a_{n j} \equiv 1(\bmod 2)\right)=2^{\sum_{i}^{n_{i}}}
$$

где $n=\sum_{i=0}^{s} n_{i} 2^{i}$ - двоичное представление числа $n, s=\left[\log _{2} n\right], n_{s}=1$.

Обозначим вектор двоичного представления числа $n$ через

$$
a(n)=\left(n_{0}, n_{1}, \ldots, n_{s}\right) .
$$

Из представления (П1) легко вывести следующие важные следствия.

$$
a\left(2^{s}\right)=(0,0, \ldots, 0,1) \Rightarrow \sum_{i} n_{i}=1 \Rightarrow \alpha\left(2^{s}\right)=2
$$

и нечетными являются лишь два крайних коэффициента.

Отметим, что только при таких значениях $n$ величина $\alpha(n)$ принимает минимальное значение, равное 2.

2)

$$
a\left(2^{s}+1\right)=(1,0, \ldots, 0,1) \Rightarrow \sum_{i} n_{i}=2 \Rightarrow \alpha\left(2^{s}+1\right)=4,
$$


и нечетными являются лишь по два крайних коэффициента $a_{n j}$ (при $j=0,1, n-1, n)$.

3) $a\left(2^{s+1}-1\right)=(1,1, \ldots, 1,1) \Rightarrow \sum_{i} n_{i}=s+1 \Rightarrow \alpha\left(2^{s+1}-1\right)=2^{s+1}=n+1$,

и, таким образом, при таких и только таких значениях $n$ все биномиальные коэффициенты - нечетные (величина $\alpha(n)$ принимает максимально возможное значение, равное $n+1)$.

4) Если $n$ - четное число $(n=2 m)$, то средний коэффициент $a_{2 m, m}$ всегда четен, так как в противном случае общее число нечетных коэффициентов было бы нечетным в противоречии с (П1).

5) В общем случае пусть $n=2^{i_{1}}+\ldots+2^{i_{j}}+2^{s}$ при некоторых $s \geq 2$, $1 \leq j \leq s, 0 \leq i_{1}<\ldots<i_{j} \leq s-1$. Тогда

$$
\sum_{i} n_{i}=j+1 \Rightarrow \alpha(n)=2^{j+1}
$$

Нечетными при этом будут лишь те коэффициенты $a_{n m}$, для которых числа $m$ имеют вид $m=\varepsilon_{i_{1}} 2^{i_{1}}+\ldots+\varepsilon_{i_{j}} 2^{i_{j}}+\varepsilon_{s} 2^{s}, \quad \varepsilon_{.}=0,1$ (их число и равно $2^{j+1}$ ).

Из этих результатов, в частности, следует, что для определенной в (16) величины $r_{n}$ справедливы оценки

$$
2^{n-1}-\frac{n+1}{2} \leq r_{n} \leq 2^{n-1}-1
$$

причем нижняя граница достигается при значениях $n$ вида $n=2^{s}-1$, а верхняя - при значениях $n$ вида $n=2^{s}$.

2. Введем еще одну характеристику треугольника биномиальных коэффициентов - число $N_{j}(s)$ значений $n$ в интервале $2^{s} \leq n \leq 2^{s+1}-1$, для которых величина $\alpha(n)=2^{j+1}$. Тогда из предыдущего следует, что

$$
N_{j}(s)=\sum_{n=2^{s}}^{2^{s+1}-1} I\left(\alpha(n)=2^{j+1}\right)=\left(\begin{array}{c}
s \\
j
\end{array}\right), \quad j=0,1, \ldots, s .
$$

Этот результат позволяет сформулировать еще одно интересное свойство биномиальных коэффициентов.

Будем обозначать символом $X(s)$ случайную величину, принимающую значения $\alpha(n)$, когда число $n$ случайно и равновероятно выбирается из множества $A(s)=\left\{2^{s}, \ldots ., 2^{s+1}-1\right\}, s \geq 1$. 
Утверждение 3. Случайная величина $X(s)$ имеет сдвинутое логбиномиальное распределение: $\log _{2} X(s)=1+\xi$, где $\xi-$ биномиальная $\operatorname{Bi}\left(s, \frac{1}{2}\right)$ случайная величина. Для ее моментов имеют место соотношения

$$
\mathbf{E} X^{k}(s)=\left(1+2^{k}\right)^{s} 2^{k-s}, \quad k=1,2, \ldots,
$$

и, в частности,

$$
\mathbf{E} X(s)=2\left(\frac{3}{2}\right)^{s}, \quad \mathbf{D} X(s)=4\left(\left(\frac{5}{2}\right)^{s}-\left(\frac{9}{4}\right)^{s}\right) .
$$

В заключение выражаем признательность А. М. Зубкову за ценные замечания, способствовавшие улучшению изложения содержания статьи.

\section{Список литературы}

1. Canteaut, A., Videau, M. Symmetric Boolean Function // IEEE Trans. Inf. Theory. - 2005. - V. 51. № 8. - P. 2791-2811.

2. Sarkar, S., Maitra, S. Efficient search for symmetric Boolean functions under constraints on Walsh spectra values // J. Comb. Math. and Comb. Comput. 2009. - V. 68. - P. 163-191.

3. Mouffron, M. Balanced alternating and symmetric functions over finite sets // Workshop on Boolean Functions: Cryptography and Applications (BFCA'08). - 2008. - Copenhagen, Denmark. - P. 27 - 44.

4. Ивченко, Г. И., Медведев, Ю. И., Миронова, В. А. Анализ спектра случайных симметрических булевых функций // Математические вопросы криптографии. - 2013. - Т. 4. Вып. 1. - С. 59 - 76.

5. Тимамев, $A$. Н. Большие уклонения в вероятностной комбинаторике. М.: Издательский дом "Академия", 2011. - 248 с.

6. Lipton, R. J., Markakis, E., Mehta, A., Vishnoi, N. K. On the Fourier spectrum of symmetric Boolean functions with applications to learning symmetric juntas // IEEE Conf. Comput. Compl. - 2005. - P. $112-119$.

7. Kolountzakis, M. N., Lipton, R. J., Markakis, E., Mehta, A., Vishnoi, N. K. On the Fourier spectrum of symmetric Boolean functions // Combinatorica. 2009. - V. 29. № 3. - P. $363-387$.

8. Lucas, E. Sur les cogruences des nombres euleriens et des coefficients differentiels des fonctions trigonometriques, suivant un module premier // Bull. Soc. Math. de France. - 1878. - V. 6. - P. $19-54$. 
